\title{
The Agriculture Vision Intelligent Image Segmentation Algorithm Based on Machine Learning
}

\author{
Deng Minghui ${ }^{1,}$, Zhu Shaopeng ${ }^{1, b}$ and Li Ming ${ }^{1, c}$ \\ ${ }^{1}$ College of Electrical and Information, Northeast Agricultural University, Harbin, China \\ amarkdmh@163.com, b luckyzhen2006@163.com, c252528907@qq.com
}

\begin{abstract}
Keywords: Image segmentation; machine vision; Random Forest
Abstract. Image segmentation and feature extraction are the premise for machine vision system to analyze and identify the image. Threshold image segmentation algorithm according to the method of two dimension threshold has a lot of calculation in calculating the threshold, and the minimum error threshold method can not use the spatial information of image. This paper presents an intelligent image segmentation algorithm with Random Forest theory based on the night segmentation and feature extraction technology. The Random Forest algorithm shows unique advantages in dealing with small sample size, high-dimensional feature space, and complex data structures. An algorithm of vision image segmentation and feature extraction based on Random Forest is designed. Experimental results show that the segmentation process of this algorithm has less control parameters and faster convergence speed.
\end{abstract}

\section{Introduction}

The normal working of any farmland production robot is dependent on the correct recognition and positioning technology of the object. Because of the complexity of the operation environment, especially the uncertainty of illumination, the similarity of environmental conditions, the individual differences and occlusion problems, the recognition and positioning technology remains to be solved. At present, we need to make use of machine vision technology, to combine other technology and to ameliorate image acquisition and image processing algorithm in order to improve the accuracy and precision of recognition and location. Machine vision technology makes use of the image sensor to acquire the image of object, converts the image to a data matrix and analyzes the image by using of computer. At the same time, a vision related task is achieved. Research and application of machine vision technique in agricultural production began in twentieth Century seventies. The study was focused on the quality inspection and classification research of peach, banana, tomato, cucumber and other agricultural products. Crop harvest automation is the application of machine vision technology in harvesting machinery and is the hottest research topic in recent years. The basic principle is that the harvest machine equipped with a camera system acquires the operation field image of farmland and woods and uses the image processing algorithm to judge whether a target image(such as fruits and vegetables). Then the harvest machine finds that target and guides the manipulator to complete the picking.

Image processing is the core technology of machine vision and mainly includes two aspects as follows:

(1) Image segmentation. Image segmentation is one of the hot spots and difficulties in image processing. The image segmentation technology segments the image into some meaningful regions and makes the feature description in these regions. We extract some features of target image and then determine whether there is the target of interest in the image.

(2) Feature extraction. The image is transformed into a more suitable for human observation or machine analysis and recognition form in order to get more useful information related to object features and objective of image processing.

Image segmentation and feature extraction are the premise for machine vision system to analyze and identify the image. Because the minimum error threshold method can not use the spatial information of image, we proposed a intelligent Random Forest algorithm in this paper to realize the 
segmentation and feature extraction of the machine vision image. The intelligent algorithm is better than the traditional segmentation algorithm and shows better effect and stability ${ }^{[1]-[8]}$.

\section{The Random Forest Algorithm}

Random forest is an ensemble training algorithm that constructs multiple decision trees. It suppresses over-fitting to the training samples by random selection of training samples for tree construction in the same way as is done in bagging, resulting in construction of a classifier that is robust against noise. Also, random selection of features to be used at splitting nodes enables fast training,even if the dimension of the feature vector is large.

Random forests have been shown to give excellent performance on a number of practical problems. They work fast, generally exhibit a substantial performance improvement over single tree classifiers such as CART, and yield generalization error rates that compare favorably to the best statistical and machine learning methods. In fact, random forests are among the most accurate general-purpose classifiers available. As a matter of fact, the statistical mechanism of random forests is not yet fully understood and is still under active investigation.

Random forests consist of ensemble of $K$ classifiers $h_{1}(x), h_{2}(x), \ldots, h_{K}(x)$. Each classifier votes for one of the classes and an instance being classified is labeled with the winning class. We denote the joint classifier as $\mathrm{h}(\mathrm{x})$. Each training set of $\mathrm{n}$ instances is drawn at random with replacement from the training set of $n$ instances. With this sampling called bootstrap replication, on average $36.8 \%$ of training instances are not used for building each tree. These out-of-bag instances come handy for computing an internal estimate of the strength and correlation of the forest.

Denote the set of out-of-bag instances for classifier $h_{K}$ as $O_{K}$. Let $Q\left(x, y_{j}\right)$ be the out-of-bag proportion of votes for class $y_{j}$ at input $\mathrm{x}$ and an estimate of $\mathrm{P}\left(\mathrm{h}(\mathrm{x})=\mathrm{y}_{\mathrm{j}}\right)$ :

$$
Q\left(x, y_{j}\right)=\frac{\sum_{k=1}^{k} I\left(h_{k}(x)=y_{j} ;(x, y) \in O_{k}\right)}{\sum_{k=1}^{k} I\left(h_{k}(x) ;\left(x, y \in O_{k}\right)\right)}
$$

where I $(\cdot)$ is the indicator function. The margin function measures the extent to which the average vote for the right class y exceeds the average vote for any other class:

$$
m r(x, y)=P(h(x)=y)-\max _{j=1, j \neq y}^{c} P\left(h(x)=y_{j}\right)
$$

It is estimated with $\mathrm{Q}(\mathrm{x}, \mathrm{y})$ and $\mathrm{Q}\left(\mathrm{x}, \mathrm{y}_{\mathrm{j}}\right)$. Strength is defined as the expected margin, and is computed as the average over the training set:

$$
S=\frac{1}{n} \sum_{i=1}^{n}\left(Q\left(x_{i}, y\right)-\max _{j=1, j \neq y}^{c} Q\left(x_{i}, y_{j}\right)\right)
$$

The average correlation is computed as the variance of the margin over the square of standard deviation of the forest:

$$
\bar{\rho}=\frac{\operatorname{var}(m r)}{s d(h())^{2}}=\frac{\frac{1}{n} \sum_{i=1}^{n}\left(Q\left(x_{i}, y\right)-\max _{j=1, j \neq y}^{c} Q\left(x_{i}, y_{j}\right)\right)^{2}-s^{2}}{\left(\frac{1}{K} \sum_{i=1}^{K} \sqrt{p_{k}+p_{k}+\left(p_{k}-p_{k}\right)^{2}}\right.}
$$

Where

$$
p_{k}=\frac{\sum_{\left(x_{i}, y\right) \in 0_{k}} I\left(h_{k}(x)=y_{j}\right)}{\sum_{\left(x_{i}, y\right) \in O_{k}} I\left(h_{k}(x)\right)}
$$

is an out-of-bag estimate of $\mathrm{P}\left(\mathrm{h}_{\mathrm{k}}(\mathrm{x})=\mathrm{y}\right)$ and 


$$
p_{k}=\frac{\sum_{\left(x_{i}, y\right) \in 0_{k}} I\left(h_{k}(x)=\dot{y}_{j}\right)}{\sum_{\left(x_{i}, y\right) \in O_{k}} I\left(h_{k}(x)\right)}
$$

is an out-of-bag estimate of $\mathrm{P}\left(\mathrm{h}_{\mathrm{k}}(\mathrm{x})=\hat{\mathrm{y}}_{\mathrm{j}}\right)$ and

$$
\dot{y}_{j}=\arg \max _{j=1, j \neq y}^{c} Q\left(x, y_{j}\right)
$$

is estimated for every instance $\mathrm{x}$ in the training set with $\mathrm{Q}\left(\mathrm{x}, \mathrm{y}_{\mathrm{j}}\right)$. The Random Forest model is shown as follows:
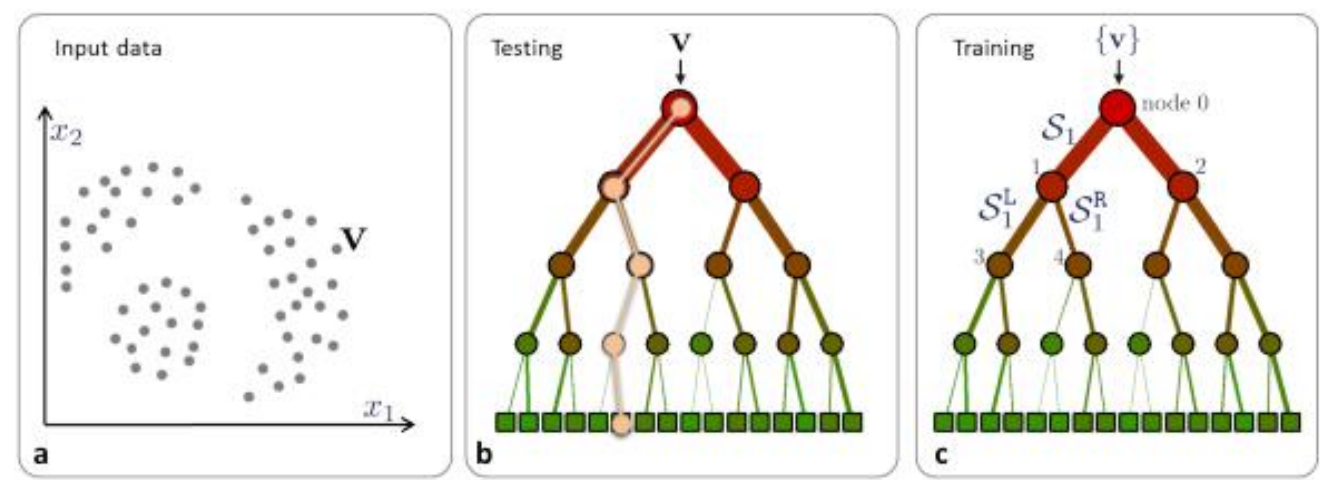

Fig.1. The Random Forest model

\section{The segmentation algorithm}

We use decision trees as base classifiers and introduces additional randomness into the trees. Namely, in each interior node of each tree a subset of $r$ attributes is randomly selected and evaluated with the index heuristics. The attribute with the highest index is chosen as split in that node. We have implemented the random forests methodology in the framework of our learning system. All presented tests were executed with the recommended values of the parameters for the forests .

The proposed method is developed on two major components: the decision forest based probabilistic classification of the agricultural vision image and propagation of region based level sets to achieve a binary segmentation of the image. The segmentation question is converted into the classification problem which is addressed by supervised random decision forest. Decision trees are discriminant classifiers which are known to suffer from over-fitting. However, a random decision forest achieves better generalization by growing an ensemble of many independent decision trees on a random subset of the training data and by randomizing the features made available at each node during training.

Due to the use of two-dimensional threshold method, the vector is selected to 2 . The independent variables represent the gray of the pixel and the rage is $[0,255]$. In the initialization, a number of particles are randomly distributed in the one-dimensional plane. The minimum error of the gray values of each particle is calculated. We find the minimum error value in the computation and then update the speed and position in accordance with the rules of Random Forest algorithm until it reaches the maximum number of iterations. The gray value of pixel corresponding with the minimum calculating error is used as the global optimal threshold for image segmentation. The process of algorithm is as follows:

(1) To initialize the number of random forest and the parameters.

(2) Training the random forest. The images are sized to a resolution of $256 \times 256$ pixels. The data consists of a collection of $9 \times 9$ neighborhood of pixels, centered at $V=(X, F)$. Where, $X=(x, y)$ denotes the position of the pixel associated with a feature vector $\mathrm{F}$. The mean and standard deviation 
of the $9 \times 9$ neighborhood are used as the feature vector F. Each tree in decision forest receives the full set $\mathrm{V}$, along with the label and the root node and selects a test to split V into two subsets to maximize information gain. A test consists of a feature and a feature response threshold. The left and the right child nodes receive their respective subsets of $\mathrm{V}$ and the process is repeated at each child node to grow the next level of the tree. Growth is terminated when either information gain is minimum or the tree has grown to maximum depth. Each decision tree in the forest is unique as each tree node selects a random subset of features and threshold. .

(3) During testing, the agricultural vision images are sized to a resolution of $256 \times 256$ before classification. Decision forests trained for each of the regions are applied to achieve a probabilistic classification of the images different regions. Computation of class posterior probabilities in decision forest is illustrated in the process.

(4) The gray value of pixel corresponding to the eventual minimum error is the optimal threshold for image segmentation.

\section{Summary}

In order to evaluate the effect of the Random Forest algorithm, the experiment still applies the standard image, as shown in Fig.1. Then we compare experiment results. Firstly, we segment two images based on the multiple kernel SVM classifier. Secondly, we segment the original image based on the Immune Genetic Algorithm and Random Forest method, results as shown in Fig. 2, Fig.3 and Fig. 4. From the experiment results, it can be shown that Random Forest algorithm has better practicability and efficiency.

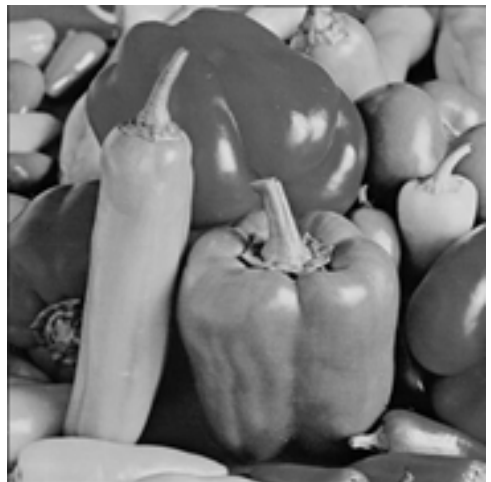

Fig.1. Test image

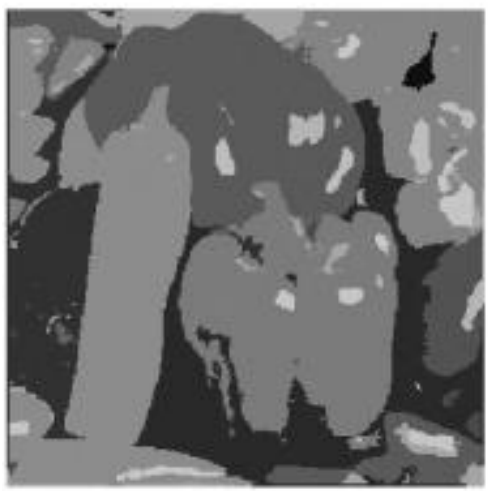

Fig.3. Immune Genetic Algorithm results

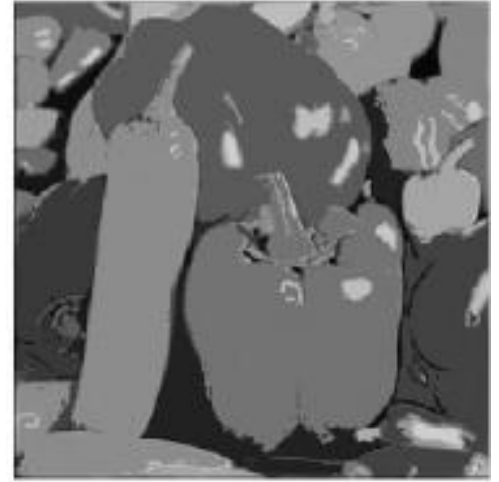

Fig2. Multiple kernel SVM classifier results

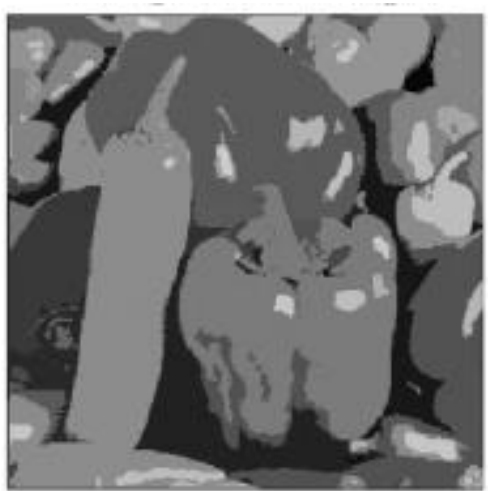

Fig.4.The Random Forest algorithm results

\section{Acknowledgments}

The paper is supported by the 2013 Natural Science Program of Heilongjiang Province Educational Committee (NO.12531011) and the Abroad Research Project of Heilongjiang Province University Strategic Reserve Talent. 


\section{References}

[1] L. Breiman. Bagging predictors. Machine Learning, 24:123 - 140, 1996.

[2] Y. Amit and D. Geman. Shape quantization and recognition with randomized trees. Neural Computation, 9:1545 - 1588, 1997.

[3] Amit, Y., Blanchard, G., \& Wilder, K. (1999). Multiple randomized classifiers: MRCL Technical Report, Department of Statistics, University of Chicago.

[4] Pal N R, Pal S K. A review on image segmentation techniques [J]. Pattern Recognition, 1993, 26:1277-1294.

[5] Y. Lin and Y. Jeon. Random forests and adaptive nearest neighbors. Journal of the American

Statistical Association, 101:578 - 590, 2006.

[6] C. Pan, D. S. Park, Y. Yang, and H. M. Yoo, "Leukocyte image segmentation by visual attention and extreme learning machine," Neural Computing and Applications, vol. 21, no. 6, pp. 1217 1227, 2012.

[7] Zhang N., Chaisattapagon C. Effective criteria for weed identification in wheat field using machine vision [J]. Transactions of the ASAE, 1995, 38(3): 135-139.

[8] Sogaard H.T., Olsen H.J, Determination of crop rows by image analysis without segmentation. Computers and Electronics in Agriculture[J]. 2003(38):141-158. 